\title{
ON LINEAR ALGEBRAIC SEMIGROUPS III
}

\author{
MOHAN S. PUTCHA
}

School of Physical and Mathematical Sciences, Department of Mathematics, North Carolina State University, Raleigh, North Carolina 27650

(Received July 18,1980)

ABSTRACT. Using some results on linear algebraic groups, we show that every connected linear algebraic semigroup S contains a closed, connected diagonalizable subsemigroup $T$ with zero such that $E(T)$ intersects each regular $J$-class of $S$. It is also shown that the lattice $(E(T), \leq)$ is isomorphic to the lattice of faces of a rational polytope in some $\mathbb{R}^{n}$. Using these results, it is shown that if $S$ is any connected semigroup with lattice of regular J-classes $U(S)$, then all maximal chains in $U(S)$ have the same length.

KEY WORDS AND PHRASES. Linear algebraic semigroup, idempotent, polytope. 1980 MATHEMATICS SUBJECT CLASSIFICATION CODES. Primary 20M10, Secondary 20699

\section{INTRODUCTION.}

Throughout this paper, $\mathbb{R}, Z, Q, \mathbb{R}^{+}, \mathrm{Z}^{+}, \mathrm{Q}^{+}$will denote the sets of reals, integers, rationals, positive reals, positive integers and positive rationals respectively. If $\mathrm{X}$ is a set then $|\mathrm{x}|$ denotes the cardinality of $\mathrm{x}$. If $\mathrm{X}$ is a subset of a semigroup, then $\langle X\rangle$ denotes the subsemigroup generated by $X$. If $(P, \leq)$ is a partially ordered set and $\left\{\alpha_{1}<\alpha_{2}<\ldots<\alpha_{n}\right\}$ is a finite chain in $P$, then we define the length of the chain to be $n-1$. K will denote a fixed algebraically closed field, $K^{n}=K \times \ldots \times K$ the affine n-space. $M_{n}(K)$ will denote the set of all $n \times n$ matrices and $G L(n, K)$ the group of units of $M_{n}(K) . F\left(X_{1}, \ldots, x_{n}\right)$ will denote the free commutative semigroup in the variables $x_{1}, \ldots, x_{n}$ and $k\left[x_{1}, \ldots, x_{n}\right]$ 
the free commutative algebra over $\mathrm{K}$ in the variables $\mathrm{X}_{1}, \ldots, \mathrm{X}_{\mathrm{n}}$. We use the notation of $[6,7]$ for algebraic semigroups. Let $S$ be an algebraic monoid with identity element $I$ and group of units $G$. If $g \in G$, then the maps $x \rightarrow x g, x \rightarrow g x$, $x \rightarrow g^{-1} x g$ are all automorphisms of the variety $S$. The last one is also a semigroup automorphism. If we let $\tilde{G}=\{(a, b) \mid a, b \in S, a b=1\}$, then $\tilde{G}$ becomes an algebraic group. Actually, with more general notions of varieties [5], G itself can be viewed as an algebraic group. By [6, Theorem 1.1], we can assume that $S$ is a closed submonoid of some $M_{n}(K)^{\prime}$. Then clearly $G=G L(n, K) \cap S$ and $S \backslash G$ is closed. If $S_{1}$ is closed submonoid of $S$ with group of units $G_{1}$, then $G_{1}=G \cap S_{1}$. If $H$ is a closed subgroup of $G$, then $H$ is the group of units of $\bar{H}$. If $S$ is connected, then clearly so is $G, \bar{G}=S$ and $\operatorname{dim} S=\operatorname{dim} G$. If $S$ is not connected, then [7; Lemma 1.9], I lies in a unique irreducible component $S_{1}$ of $S$ and $S_{1}$ is a closed connected submonoid of $S$. We say that $S$ is trigonalizable if $S$ is *-isomorphic to a closed semigroup of lower triangular matrices. If $\mathrm{S}$ is connected, then since $\bar{G}=S$, it follows from the Lie-Kolchin Theorem [5; Theorem 17.6] that $S$ is trigonalizable if and only if $G$ is solvable. $S$ is a d-semigroup if $S$ is *-isomorphic to a closed subsemigroup of $\left(\mathrm{K}^{\mathrm{p}}, \cdot\right)$ for some $\mathrm{p} \in \mathrm{Z}^{+}$. If $\mathrm{S}$ is connected, then since $\bar{G}=S$, we see that $S$ is a d-semigroup if and only if $G$ is a torus. By [7; Corollary 3.15], a connected d-semigroup with zero can be characterized as a connected Clifford semigroup with zero. If $X, Y \subseteq S$, then $X$ is conjugate to $Y$ if $g^{-1} X g=Y$ for some $g \in G$.

\section{CONNECTED SEMIGROUPS}

LEMMA 1.1. Let $S$ be a connected monoid, $e \in E(S), e \neq 1$. Then there exists a closed connected submonoid $S^{\prime}$ of $S$ such that 1 , e $\in S^{\prime}$ and $e$ is the zero of $S^{\prime}$. PROOF. Let $G$ denote the group of units of $\mathrm{S}$ and set $\mathrm{V}=\mathrm{S} \backslash \mathrm{G}$. Then $\mathrm{V}=$ $v_{1} \cup \ldots \cup v_{r}$ where $v_{1}, \ldots, v_{r}$ are closed and irreducible. Let $m_{i}=\operatorname{dim} v_{i}$, $i=1, \ldots, r$. Then $m_{i} \leq n-1$, where $n=\operatorname{dim} S$. Let $\phi: S \rightarrow$ eS be given by $\phi(x)=$ ex. Let $q=\operatorname{dim} e S, \phi_{i}=\left.\phi\right|_{V_{i}}, w_{i}=\overline{\phi\left(V_{i}\right)} \subseteq$ eS. Let $i \in\{1, \ldots, r\}$. Suppose $w_{i}=$ eS. Then $\phi_{i}$ is dominant. So by [5; Theorem 4.3], there exists a non-empty open set $0_{i}$ of eS such that $0_{i} \subseteq \phi\left(v_{i}\right)$ and so that $\operatorname{dim} \phi_{i}^{-1}(x)=m_{i}-q<n-q$ for all $x \in 0_{i}$. So 


$$
\operatorname{dim}\left(v_{i} \cap \phi^{-1}(x)\right)<n-q \text { for all } x \in 0_{i}
$$

Next suppose $w_{i} \neq$ eS. Then set $o_{i}=$ eSl $w_{i}$. Then

$$
v_{i} \cap \phi^{-1}(x)=\emptyset \text { for all } x \in 0_{i} .
$$

Let $0=0_{1} \cap O_{2} \cap \ldots \cap O_{r}$. Since eS is connected, $0 \neq \varnothing$. Let $x \in 0$. Then $x \in \phi^{-1}(x)$. Let $D$ be an irreducible component of $\phi^{-1}(x)$ such that $x \in D$. Then [5; Theorem 4.1] $\operatorname{dim} D \geq n-q$. We claim that $D \nsubseteq V$. For suppose $D \subseteq V$. Then $D \subseteq v_{i}$ for some $i$. Since $x \in \phi^{-1}(x) \cap 0_{i} \cap v_{i}$, (2) is ruled out. So by (I) $\operatorname{dim} \mathrm{D}<\mathrm{n}-\mathrm{q}$, a contradiction. Hence $\mathrm{D} \nsubseteq \mathrm{V}$. So $\mathrm{D} \cap \mathrm{G} \neq \varnothing$. Let $g \in \mathrm{D} \cap \mathrm{G}$. So $\phi(g)=x$. Thus eg $=x, \mathrm{xg}^{-1}=\mathrm{e}$. Let $\mathrm{Y}=\mathrm{Dg}^{-1}$. Then $\mathrm{Y}$ is closed and irreducible. Let $\mathrm{y} \in \mathrm{Y}$. Then $\mathrm{yg} \in \mathrm{D}$. So eyg $=\mathrm{x}$ and $\mathrm{ey}=\mathrm{xg}^{-1}=\mathrm{e}$. Hence ey $=\mathrm{e}$ for all $y \in Y$. Since $g \in D, I=g g^{-1} \in Y$. Since $x \in D, e=x g^{-1} \in Y$. Let $S_{1}=\{a \mid a \in S$, ea $=e\}$. Then $S_{1}$ is a closed submonoid of $S$ and $Y \subseteq S_{1}$. Let $S_{2}$ be the (unique) irreducible component of 1 in $S_{1}$. Then $Y \subseteq S_{2}$ and $S_{2}$ is a closed connected submonoid of $\mathrm{S}$. Thus $\mathrm{I}$, e $\in \mathrm{S}_{2}$ and ea $=\mathrm{e}$ for $\mathrm{a} \in \mathrm{S}_{2}$. By the dual of the above argument, there exists a closed connected submonoid $\mathrm{S}_{3}$ of $\mathrm{S}_{2}$ such that $e \in S_{3}$ and $a e=e$ for all $a \in S_{3}$. So ae $=e a=e$ for all $a \in S_{3}$.

FACT 1.2. Let $A \subseteq M_{n}(K)$ such that $A B=B A$ for all $A, B \in A$. Suppose also that each $A \in A$ is lower triangular and diagonalizable. Then there exists a lower triangular, invertible matrix $P$ such that $P^{-1} A P$ is diagonal.

PROOF. We prove by induction on $n$. Let $A=\left\{A_{\alpha} \mid \alpha \in \Omega\right\}, A_{\alpha}=\left[\begin{array}{ll}a_{\alpha} & 0 \\ B_{\alpha} & C_{\alpha}\end{array}\right]$. is $(n-1) \times(n-1), a_{\alpha} \in K$. Clearly $C_{\alpha} C_{\beta}=C_{\beta} C_{\alpha}$ for all $\alpha, \beta$. Since

$$
\text { minimum polynomial of } \mathrm{C}_{\alpha} \mid \text { minimum polynomial of } \mathrm{A}_{\alpha} \text {, }
$$

minimum polynomial of $\mathrm{C}_{\alpha}$ has no multiple roots. So each $\mathrm{C}_{\alpha}$ is diagonalizable. So there exists, by induction, an invertible, lower triangular $(n-1) \times(n-1)$ matrix $M_{1}$ such that $M_{1}^{-1} C_{\alpha} M_{1}$ is diagonal for all $\alpha$. Let $M=\left[\begin{array}{ll}1 & 0 \\ 0 & M_{1}\end{array}\right]$. Then 


$$
D_{\alpha}=M^{-1} A_{\alpha} M=\left[\begin{array}{ll}
a_{\alpha} & 0 \\
F_{\alpha} & G_{\alpha}
\end{array}\right], \quad G_{\alpha} \text { is }(n-1) \times(n-1) \text { and diagonal. }
$$

Let $E_{\alpha}=D_{\alpha}-a_{\alpha} I, \quad \alpha \in \Omega$. Then each $E_{\alpha}$ is diagonalizable and $E_{\alpha} E_{\beta}=E_{\beta} E_{\alpha}$ (all $\alpha, \beta)$. Moreover,

$$
E_{\alpha}=\left[\begin{array}{lll}
0 & 0 & \\
b_{2}^{(\alpha)} & c_{2}^{(\alpha)} & 0 \\
\cdot & \cdot & \\
\cdot & 0 & \cdot \\
b_{n}^{(\alpha)} & & c_{n}^{(\alpha)}
\end{array}\right]
$$

Since $E_{\alpha} E_{\beta}=E_{\beta} E_{\alpha}$,

$$
c_{i}^{(\alpha)} b_{i}^{(\beta)}=b_{i}^{(\alpha)} c_{i}^{(\beta)}, \quad i=2, \ldots, n, a l l \alpha, \beta
$$

Also, since $\mathrm{E}_{\alpha}$ is diagonalizable,

$$
c_{i}^{(\alpha)}=0 \text { implies } b_{i}^{(\alpha)}=0 \text {, all } i, \alpha
$$

Let $i \in\{2, \ldots, n\}$. If there exists $\alpha$ such that $c_{i}^{(\alpha)} \neq 0$, let $u_{i}=-b_{i}^{(\alpha)} / c_{i}^{(\alpha)}$. By (3), $u_{i}$ is independent of the choice of $\alpha$. If there is no such $\alpha$, let $u_{i}=0$. Let $u_{1}=1$ and set $u=\left[\begin{array}{c}u_{1} \\ \vdots \\ \dot{u}_{n}\end{array}\right]$. By $(4), E_{\alpha} u=0$ for all $\alpha$. Let $e_{i}$ be the column with $I$ in $i^{\text {th }}$ component and 0 elsewhere. Then $u, e_{2}, \ldots, e_{n}$ is a linearly independent set of eigenvectors of $E_{\alpha}$ for all $\alpha \in \Omega$. Let $R=\left[u, e_{2}, \ldots, e_{n}\right]$. Then $R$ is lower triangular and invertible. Clearly $R^{-1} E_{\alpha} R$ is diagonal for all $\alpha$. So $R^{-I} D_{\alpha} R$ is diagonal for all $\alpha$. Let $P=M R$.

LEMMA 1.3. Let $\mathrm{S}$ be a connected monoid with identity element 1 , zero e. Let $G$ denote the group of units of $S$. Suppose $G$ is solvable. Then for any maximal torus $T$ of $G, e \in \bar{T}$.

PROOF. We can assume that $S$ is a closed submonoid of $M_{n}(K)$. By the LieKolchin theorem [5; Theorem 17.6] there exists $P \in G L(n, K)$ such that $P^{-1}$ GP is lower triangular. Since $\bar{G}=S, P^{-1} S P$ is lower triangular. So we can assume 
that $\mathrm{S}$ is lower triangular. Let $\mathrm{T}$ be a maximal torus of $\mathrm{G}$ and $\operatorname{set} \mathrm{X}=\mathrm{T} U\{\mathrm{e}$. Then X satisfies the hypothesis of Fact 1.2. So there exists a lower triangular $R \in G L(n, K)$ such that $R^{-1} X R$ is diagonal. Clearly $R^{-1} S R$ remains lower triangular. So we can assume that $X$ is diagonal. If $a \in S$, then let $\phi(a)$ be the $n \times n$ diagonal matrix, with the diagonal being that of a. Then $\phi(X)=X$. Clearly $\phi$ is a *-homomorphism of $S$ into $M_{n}(K)$ and $\phi(G)$ is a torus. By [5; Corollary 2l.3C], $\phi(G)=\phi(T)=T$. So $\phi(G) \subseteq S$. Since $\bar{G}=S, \phi(S) \subseteq S$. Let $W=\phi(S)$. Then $W=\{a \mid a \in S, \phi(a)=a\}$ is closed. Since $\phi(S)=W, W$ is a closed connected submonoid of $\mathrm{S}$. Let $\mathrm{H}$ denote the group of units of $\mathrm{W}$. Then $\mathrm{T} \subseteq \mathrm{H} \subseteq \mathrm{G}$ and $\mathrm{H}$ is a torus. So $T=H$ and $W=\bar{T}$. Clearly $e=\phi(e) \in W=\bar{T}$.

THEOREM 1.4. Let $\mathrm{S}$ be a connected monoid with group of units $\mathrm{G}$. Let $\mathrm{B}$ be a Borel subgroup of $G$. Then $S=\underset{x \in G}{\cup} x \bar{B}^{-1}$.

PROOF. We can assume that $S$ is a closed submonoid of $W=M_{n}(K)$. Let ${ }_{{ }_{1}}=\left\{\left(a, a^{-1}\right) \mid a \in G\right\} \quad$ Then $G_{1}$ is a closed subset of $W \times W$. If $(a, b),(c, d) \in G$, then define $(a, b)(c, d)=(a c, d b)$. Then $G_{I}$ is an algebraic group *-isomorphic to G. Let $B_{1}=\left\{\left(a, a^{-1}\right) \mid a \in B\right\}$. Then $B_{1}$ is a Borel subgroup of $G_{1}$. Now [5; Theorem 2l.3], $G_{1} / B_{1}$ is a projective variety. Let $\phi: G_{1} \rightarrow G_{1} / B_{1}$ be the natural projection $\phi(a)=a B_{1}$. Let $V=W \times G_{1}, Y=W \times G_{1} / B_{1}$. By [1; Theorem 6.8], $G_{1} / B_{1}$ is smooth and hence a normal variety. The same is true for $W$. So $Y=W \times G_{1} / B_{1}$ is normal $[1 ; \mathrm{p} .77]$. Let $\psi: \mathrm{V} \rightarrow \mathrm{Y}$ be given by $\psi(\mathrm{a}, \mathrm{b})=(\mathrm{a}, \phi(\mathrm{b}))$. Then $\psi$ is a surjective morphism. Clearly each fibre of $\psi$ is irreducible and has dimension equal to that of $B_{1}$. So [1; Proposition 18.4], $\psi$ is an open map. Let $X=\left\{\left(a, g, g^{-1}\right) \mid a \in S\right.$, $\left.g \in G, g^{-1} a g \in \bar{B}\right\}$. Then $X$ is closed in $V$. So $\psi(\sim X)$ is open in $Y$. Hence $\sim \psi(\sim X)$ is closed in $Y$. Clearly $\sim \psi(\sim X) \subseteq \psi(X)$. Suppose $\omega \in \psi(X), \omega \in \psi(\sim X)$. Then $\omega=\psi(x)=\psi(y)$ for some $x \in X, y \in \sim X$. So $x=\left(a, g, g^{-1}\right), y=\left(a, h, h^{-1}\right)$ for some $a \in S, g, h \in G$. Now $g^{-1} a g \in \bar{B}$. Since $\psi(x)=\psi(y), \phi\left(g, g^{-1}\right)=\phi\left(h, h^{-1}\right)$. So $g B=h B$. Thus $h=g b$ for some $b \in B$. So $h^{-1} a h=b^{-1}\left(g^{-1} a g\right) b \in b^{-1} \overline{B b}=\bar{B}$, $a$ contradiction. So $\sim \psi(\sim X)=\psi(X)$ and $\psi(X)$ is closed. Let $\theta: Y=W \times G_{1} / B_{I} \rightarrow W$ denote the projection of $Y$ onto $W$. Then since $G_{I} / B_{I}$ is projective, $\theta$ is a closed 
map [5; Theorem 6.2]. Hence $\theta(\psi(x))$ is closed in W. Clearly $\theta(\psi(x))=$

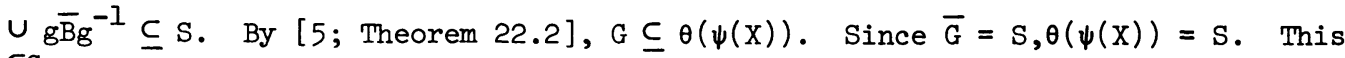
$g \in G$

proves the theorem.

COROLLARY 1.5. Let $\mathrm{S}$ be a connected monoid with zero e and $\mathrm{T}$ a maximal torus in the group of units $G$ of $S$. Then $e \in \bar{T}$.

PROOF. Now $T \subseteq B$ for some Borel subgroup $B$ of $G$. By Theorem 1.4, e $\in \times \bar{B}^{-1}$ for some $x \in G$. So $e=x^{-1} e x \in \bar{B}$. Hence $e$ is the zero of $\bar{B}$. By Lemma $1.3, e \in \bar{T}$.

COROLLARY 1.6. Let $S$ be a connected monoid with group of units $G$. Let $e_{1}, \ldots, e_{k} \in E(S)$ such that $e_{1}>e_{2}>\ldots>e_{k}$. Then there exists a maximal torus $\mathrm{T}$ of $\mathrm{G}$ such that $\mathrm{e}_{1}, \ldots, \mathrm{e}_{\mathrm{k}} \in \overline{\mathrm{T}}$.

PROOF. We prove by induction on $k$. If $k=1$, we are done by Lemma 1.1 and Corollary 1.5. So assume $\mathrm{k}>1$. By Lemma 1.1, there exist closed connected submonoids $s_{l}, \ldots, s_{k}$ of $S$ such that $e_{i}$ is the zero of $s_{i}$. Then $a e_{k}=e_{k} a=e_{k}$ for all $a \in S_{i}, i=1, \ldots, k$. Let $V=\left\{a \mid a \in S, a e_{k}=e_{k} a=e_{k}\right\}$. Then $V$ is a closed submonoid of $\mathrm{S}$ and $\mathrm{S}_{1}, \ldots, \mathrm{S}_{\mathrm{k}} \subseteq \mathrm{V}$. Let $\mathrm{W}$ be the (unique) irreducible component of $I$ in $V$. Then $S_{1}, \ldots, S_{k} \subseteq W$ and $W$ is a closed connected submonoid of $S$. So $e_{k}$ is the zero of $S$. Let $G_{1}$ denote the group of units of $W$. By our induction hypothesis, there exists a maximal torus $T_{1}$ and $G_{1}$ such that $e_{1}, \ldots, e_{k-1} \in \bar{T}_{1}$. By Corollary 1.5, $e_{k} \in \bar{T}_{1}$. Let $T_{1} \subseteq T$ where $T$ is a maximal torus of $G$. Then $e_{1}, \ldots, e_{k} \in \bar{T}$. By [7; Lemma 1.3] we have,

LEMMA 1.7. Let $\mathrm{S}$ be a semigroup, $\mathrm{J}_{1}, \ldots, \mathrm{J}_{\mathrm{k}} \in U(\mathrm{~S}), \mathrm{J}_{1}>\mathrm{J}_{2}>\ldots>\mathrm{J}_{\mathrm{k}}$. Then there exists $e_{1}, \ldots, e_{k} \in E(S)$ such that $e_{i} \in J_{i}, i=1, \ldots, k$ and $e_{1}>e_{2}>\ldots>e_{k}$

THEOREM 1.8. Let $\mathrm{S}$ be a connected monoid with group of units $\mathrm{G}$. Then

(1) All maximal closed connected d-submonoids of $\mathrm{S}$ are conjugate.

(2) All maximal closed connected d-submonoids with zeroes, of S, are conjugate.

(3) Let $Y$ be a maximal closed connected d-submonoid with zero, of $S$. Then $U \mathrm{gE}(\mathrm{Y}) \mathrm{g}^{-1}=\mathrm{E}(\mathrm{S})$. In particular $E(Y) \cap \mathrm{J} \neq \emptyset$ for all $\mathrm{J} \in U(\mathrm{~S})$. More$g \in G$ 
PROOF. Since the group of units of a maximal closed connected d-submonoid of $S$ is a maximal torus in $G$, (1) follows from [5; Corollary 21.3A]

(2) Let $S_{1}, S_{2}$ be two maximal closed connected d-submonoids with zeroes of S. Let $e_{i}$ be the zero of $S_{i}, i=1,2$. Let $H_{i}$ be the group of units of $S_{i}$, $i=1,2$. Then $H_{i} \subseteq T_{i}, T_{i}$ a maximal torus of $G, i=1,2$. Let $v_{i}=\bar{T}_{i}, i=1,2$. Let $f_{i}$ be the minimum idempotent of $v_{i}$. Then $e_{i} \geq f_{i}$. Let $w_{i}=\left\{a \mid a \in v_{i}\right.$, af $\left.f_{i}=f_{i}\right\}, U_{i}$ the (unique) irreducible component of 1 in $w_{i}$. Since $v_{1}, v_{2}$ are conjugate by $(1)$, so are $W_{1}, W_{2}$. Hence $U_{1}, U_{2}$ are conjugate. Since $S_{i} \subseteq W_{i}$, $S_{i} \subseteq U_{i}, i=1,2$. By Lemma 1.1, $f_{i} \in U_{i}, i=1,2$. By the maximality of $s_{i}$, $S_{i}=U_{i}, \quad i=1,2$.

(3) Let $e \in E(S)$. By Corollary 1.6, e $\in S_{1}$ for some closed connected dsubmonoid $S_{1}$ of $S$. By [7; Theorem 3.16], there exists a closed connected dsubmonoid with zero, $S_{2}$ of $S_{1}$ such that $e \in S_{2}$. By (2) $x_{2} x^{-1} \subseteq Y$ for some $x \in G$. So $\operatorname{xex}^{-1} \in Y$. Hence $E(Y) \cap J_{e} \neq \emptyset$. Next let $J_{I}>J_{2}>\ldots>J_{k}$ be a maximal chain in $U(S)$. By Lemma 1.7 , there exist $e_{i} \in E\left(J_{i}\right)$ such that $e_{1}>e_{2}>\ldots>e_{k} \cdot$ Clearly

$$
e_{1}>e_{2}>\ldots>e_{k}
$$

is a maximal chain in $E(S)$. For if $e_{i}>f>e_{i+1}, f \in E(S)$, then $J_{e_{i}}>J_{f}>J_{e_{i+1}}$, a contradiction. By Corollary 1.6, $e_{1}, \ldots, e_{k} \in M_{1}$ for some closed connected dsubmonoid $M_{1}$ of $S$. By [7; Theorem 3.16], $e_{1}, \ldots, e_{k} \in M_{2}$ for some closed connected d-submonoid with zero, $M_{2}$ of $M_{1}$. So $e_{1}, \ldots, e_{k} \in M_{3}$ for some maximal connected d-submonoid with zero, $M_{3}$ of $S$. Since (5) is maximal in $E(S)$, it is maximal in $E\left(M_{3}\right)$. By [7; Theorem 3.17], $\operatorname{dim} M_{3}=k-1$. By (2) $\operatorname{dim} M_{3}=\operatorname{dim} Y$.

THEOREM 1.9. Let $S$ be a connected semigroup. Then all maximal chains in $U(\mathrm{~S})$ have the same length.

PROOF. $U(S)$ has maximum element $J_{0}$. Let $e \in E\left(J_{0}\right)$. By $[7 ;$ Lemma 1.3, 1.7]. $U($ eSe $)=\{J \cap$ eSe $\mid J \in U(S)\} \simeq U(S)$. Now eSe is a connected monoid. We are done by Theorem $1.8(3)$.

THEOREM 1.10. Let $S$ be $a$ connected monoid such that for $a$ ll $a, b \in S, a \mid b$ 
implies $a^{2} \mid b^{i}$ for some $i \in z^{+}$. Let $Y$ be a maximal closed connected d-submonoid with zero, of $S$. Then $J \cap Y$ is a subgroup of $Y$ for all $J \in U(S)$. In particular $U(Y)=\{J \cap Y \mid J \in U(6)\}$ and $(U(S), \leq) \cong(E(Y), \leq)$.

PROOF. The hypothesis implies by [8] that $J$ is a subsemigroup of $S$ for all $J \in U(S)$. Let $J \in U(S)$. Then $J \cap Y \neq \emptyset$ by Theorem 1.8. Let $a, b \in J \cap Y$. Then atle, $b H f$ in $Y$ for some e, $f \in E(Y)$. So $e, f \in J$. Since e, $f \in Y$, ef $=f e$. Since $J$ is completely simple, $e=f$. So attb in $Y$ and $J \cap Y$ is a subgroup of $Y$.

Now applying the proof of Theorem 1.9 we have,

COROLLARY 1.11. Let $\mathrm{S}$ be a connected semigroup such that for $\mathrm{all} \mathrm{a}, \mathrm{b} \in \mathrm{S}$, $a \mid b$ implies $a^{2} \mid b^{i}$ for some $i \in z^{+}$. Then $(U(S), \leq) \cong(E(Y), \leq)$ for some connected d-monoid with zero, Y.

THEOREM 1.12. Let $S$ be a connected monoid such that the group of units $G$ of $S$ is nilpotent. Then $E(S)$ is finite.

PROOF. By [5; Proposition 19.2], G has a unique maximal torus T. So $\bar{T}$ is the unique maximal closed connected d-submonoid of $\mathrm{S}$. By Theorem 1.8, $\mathrm{E}(\mathrm{S}) \subseteq \overline{\mathrm{T}}$. So $E(S)$ is finite.

EXAMPLE 1.13. $S=\left\{\left[\begin{array}{lll}a & b & c \\ 0 & a & d \\ 0 & 0 & a\end{array}\right] \mid a, b, c, d \in K\right\}$ is an example of a connected monoid satisfying the hypothesis of Theorem 1.12.

CONJECTURE 1.14. Let $S$ be a connected monoid with zero such that $E(S)$ is finite. Then the group of units of $S$ is solvable.

EXAMPLE 1.15. Let $S=\left\{\left[\begin{array}{ll}a & b \\ 0 & a^{2}\end{array}\right] \mid a, b \in S\right\}$. Then $S$ is a connected monoid with zero and $|E(S)|=2$. The group of units of $S$ is solvable but not nilpotent.

\section{CONNECTED A-SEMIGROUPS WITH ZEROS}

Let $S$ be a connected d-semigroup with zero, $\operatorname{dim} S>0$. Then $S$ is a monoid [7; Theorem 2.7]. By a character of $S$, we mean $a *$-homomorphism $X: S \rightarrow K$ such that $X(I)=I, X(0)=0$. We let $\Phi(S)$ denote the commutative semigroup of all characters of $\mathrm{S}$ with pointwise multiplication. It is clear that if $\mathrm{S}_{1}, \mathrm{~S}_{2}$ are connected $\mathrm{d}-$ 
semigroups with zeros, $\operatorname{dim} S_{i}>0$, then $S_{1}{ }^{*}$-isomorphic to $S_{2}$ implies $\Phi\left(S_{1}\right) \cong \Phi\left(S_{2}\right)$.

A commutative semigroup $W$ is said to be totally cancellative if $W$ is cancellative and for all $a, b \in W, n \in z^{+}, a^{n}=b^{n}$ implies $a=b$. We will need the following result of Grillet [3; Theorem 2.2].

THEOREM A [Gillet]. Let $\mathrm{W}$ be a finitely generated commutative semigroup. Then $\mathrm{W}$ can be embedded in a free commutative semigroup if and only if $\mathrm{W}$ is totally cancellative and idempotent-free.

LEMMA 2.1. Let $\mathrm{S}$ be a connected d-semigroup with zero 0 and identity 1 , $\operatorname{dim} S>0 . \quad$ Then

(1) $\Phi(S) \neq \varnothing$.

(2) If $e \in E(S)$, e $\neq 0$, then there exists $x \in \Phi(S)$ such that for all $g \in E(S)$, $g \geq$ e implies $\chi(g)=1, g \not$ e implies $\chi(g)=0$.

(3) $\Phi(S)$ is idempotent-free and totally cancellative.

(4) $\Phi(S)$ is linearly independent in the vector space of all functions from $\mathrm{S}$ into $\mathrm{K}$.

PROOF. Let $G$ denote the group of units of $S$. We can assume that $S$ is a closed submonoid of $M_{n}(K)$ for some $n \in z^{+}$. If $a \in S$, let $\alpha(a)=\operatorname{det} a$. Then $\alpha \in \Phi(S), \quad \alpha(f)=0$ for $f \in E(S), f \neq 1$. So $\Phi(S) \neq \varnothing$. Let e $\in E(S)$, e $\neq 0$. Then by the above, there exists $\beta \in \Phi(e S)$ such that $\beta(f)=0$ for all $f \in E(e S)$ with $f \neq e$. Define $\chi: S \rightarrow K$ as $\chi(a)=\beta($ ea). This proves (2).

Let $x \in \Phi(S)$ such that $\chi^{2}=x$. Then $\chi(S)=\{1,0\}$ contradicting the fact that $S$ is connected. So $\Phi(S)$ is idempotent-free. Let $x_{1}, x_{2}, x_{3} \in \Phi(S)$ such that $x_{1} x_{2}=x_{1} x_{3}$. If $a \in G$, then $x_{1}(a) \neq 0$ and so $x_{2}(a)=x_{3}(a)$. So $x_{2}=x_{3}$ on $G$. Since $\bar{G}=S, x_{2}=x_{3}$ on $S$. So $\Phi(S)$ is cancellative. Now let $x_{1}, x_{2} \in \Phi(S)$, $\mathrm{m} \in \mathrm{z}^{+}$such that $\mathrm{X}_{1}^{\mathrm{m}}=\mathrm{X}_{2}^{\mathrm{m}}$. Let $\mathrm{Y}=\left\{\xi \mid \xi \in \mathrm{K}, \xi^{\mathrm{m}}=1\right\}$. Then $\mathrm{Y}$ is finite. If $\xi \in Y$, let $S_{\xi}=\left\{a \mid a \in S, X_{1}(a)=\xi X_{2}(a)\right\}$. If $a \in G$, then $X_{i}(a) \neq 0$ for $i=1,2$. So $a \in S_{\xi}$ for some $\xi \in Y$. Thus $G \subseteq \underset{\xi \in Y}{\cup} S_{\xi}$. Since $\bar{G}=S, S=\underset{\xi \in Y}{U} S_{\xi}$. Since $S$ is connected, $\mathrm{S}=\mathrm{S}_{\xi}$ for some $\xi \in \mathrm{Y}$. In particular, $1=\mathrm{X}_{1}(1)=\xi \mathrm{X}_{2}(1)=\xi$. So $x_{1}=x_{2}$ and $\Phi(S)$ is totally cancellative. This proves (3). 
Now let $x_{1}, \ldots, x_{m} \in \Phi(S)$ be distinct characters of $S$ which are linearly dependent. Let $\psi_{i}$ denote the restriction of $x_{i}$ to $G$. Then $\psi_{i}, i=1, \ldots, m$ are linearly dependent homomorphism of G. So [5; Lemma 16.1], $\psi_{i}=\psi_{j}$ for some $i \neq j$. Since $\bar{G}=S, x_{i}=x_{j}$, contradiction. This proves the lemma.

LEMMA 2.2. Let $S$ be a connected d-semigroup with zero, dim $S>0$. Then

(1) $S$ is *-isomorphic to a closed submonoid $S^{\prime}$ of $\left(K^{n}, \cdot\right)$ for some $n \in Z^{+}$ such that $0=(0, \ldots, 0) \in S^{\prime}$.

(2) $\Phi(S)$ is finitely generated.

(3) If $a, b \in S, s \neq b$, then there exists $x \in \Phi(S)$ such that $x(a) \neq x(b)$.

PROOF. First we prove (I). We can assume that $S$ is closed subsemigroup of $\left(K^{n}, \cdot\right)$ for some $n \in z^{+}$, $n$ minimal. Let $e$ denote the zero of $S$ and set $S_{1}=$

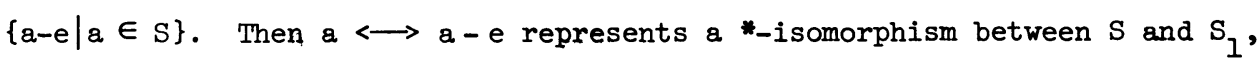
$0=(0, \ldots, 0)$ is the zero of $S_{1}$. So without loss of generality we can assume that $e=(0, \ldots, 0)$. Let $f=\left(\alpha_{1}, \ldots, \alpha_{n}\right)$ denote the identity of $s$. So $\alpha_{i}^{2}=\alpha_{i}$, $i=1, \ldots, n$. Suppose some $\alpha_{i}=0$, say $i=1$. Then $n>1$ and $S \subseteq\{0\} \times K^{n-1}$. So $S$ is *-isomorphic to a closed subsemigroup of $\left(K^{n-1}, \cdot\right)$, contradicting the minimality of $n$. So $\alpha_{i} \neq 0, i=1, \ldots, n$. So $\alpha_{i}=1, i=1, \ldots, n$ and $S$ is a closed submonoid of $\left(K^{n}, \cdot\right)$. This proves (1).

Let $S$ be a closed submonoid of $\left(K^{n}, \cdot\right)$ with identity $1=(1, \ldots, 1)$, zero $0=(0, \ldots, 0)$. Let $x_{i}$ denote the $i^{\text {th }}$ projection of $s$ into $K$. Then clearly $x_{1}, \ldots, x_{n} \in \Phi(S)$. Let $x \in \Phi(S)$. Since $x(0)=0, x$ does not have a constant term. So there exist $\omega_{1}, \ldots, \omega_{t} \in F\left(x_{1}, \ldots, x_{n}\right), \alpha_{1}, \ldots, \alpha_{n} \in K$ such that

$$
x(a)=\sum_{i=1}^{t} \alpha_{i} \omega_{i}(a) \text { for all } a \in S \text {. }
$$

So $x=\sum_{i=1}^{t} \alpha_{i} \omega_{i}\left(x_{1}, \ldots, x_{n}\right)$. By Lemma 2.1 (4), $x=\omega_{i}\left(x_{1}, \ldots, x_{n}\right)$ for some $i$. So $\Phi(S)=\left\langle x_{1}, \ldots, x_{n}\right\rangle$. This proves (2). Let $a, b \in S$ such that $x(a)=x(b)$ for all $x \in \Phi(S)$. Then $a=\left(x_{1}(a), \ldots, x_{n}(a)\right)=\left(x_{1}(b), \ldots, x_{n}(b)\right)=b$. This proves (3).

LEMMA 2.3. Let $S$ be a closed connected submonoid of $\left(K^{n}, \cdot\right)$ with zero $0=(0, \ldots, 0)$. Then there exist $u_{1}, \ldots, u_{t}, v_{1}, \ldots, v_{t} \in F\left(x_{1}, \ldots, x_{n}\right)$ such that for 
$a \in K^{n}, a \in S$ if and only if $u_{i}(a)=v_{i}(a), i=1, \ldots, t$.

PROOF. Let $x_{i}$ denote the $i^{\text {th }}$ projection of $s$ into $K$. Then $x_{1}, \ldots, x_{n} \in \Phi(S)$. Let $I=\left\{f \mid f \in K\left[X_{1}, \ldots, X_{n}\right], f(S)=0\right\}$. Let $D=\{f \mid f \in I, f=u-v$ for some $u$, $\left.v \in F\left(x_{1}, \ldots, x_{n}\right)\right\}$. We claim that $I=(D)$. Suppose not. Then there exists $f \in I$, $f \notin(D)$. Since $f(0)=0$, there exist $\omega_{1}, \ldots, \omega_{r} \in F\left(X_{1}, \ldots, X_{n}\right), \alpha_{1}, \ldots, \alpha_{r} \in K \backslash\{0\}$ such that $f=\sum_{i=1}^{r} \alpha_{i} \omega_{i}$. Of all such $f$ choose one with $r$ minimal. So $\sum_{i=1}^{r} \alpha_{i} \omega_{i}(a)=$ $f(a)=0$ for all $a \in S$. So

$$
\sum_{i=1}^{r} \alpha_{i} \omega_{i}\left(x_{1}, \ldots, x_{n}\right)=0
$$

By Lemma $2.1(4), \omega_{p}\left(x_{1}, \ldots, x_{n}\right)=\omega_{q}\left(x_{1}, \ldots, x_{n}\right)$ for some $p, q \in\{1, \ldots, r\}, p \neq q$. Assume $p=1, q=2$. So $\omega_{1}(a)=\omega_{2}(a)$ for all $a \in S$. Thus $\omega_{1}-\omega_{2} \in$ D. Now

$$
\left(\alpha_{1}+\alpha_{2}\right) \omega_{2}+\sum_{i=3}^{r} \alpha_{i} \omega_{i}=f-\alpha_{1}\left(\omega_{1}-\omega_{2}\right) \in I
$$

By minimality of $r, f-\alpha_{1}\left(\omega_{1}-\omega_{2}\right) \in(D)$. So $f \in(D)$, a contradiction. Thus $I=(D)$. By the Hilbert Basis Theorem there exist $f_{1}, \ldots, f_{t} \in I$ such that $I=\left(f_{1}, \ldots, f_{t}\right)$. Since $I=(D), f_{1}, \ldots, f_{t} \in\left(D_{1}\right)$ for some finite subset $D_{1}$ of $D$. So $\left(D_{1}\right)=I$. This proves the lemma.

Let $\mathrm{S}_{1}, \mathrm{~S}_{2}$ be connected d-semigroups with zeros, $\operatorname{dim} \mathrm{S}_{i}>0, i=1$, 2 . Let $\phi: S_{1} \rightarrow S_{2}$ be a *-homomorphism such that $\phi(1)=1, \phi(0)=0$. Then define $\phi^{*}: \Phi\left(S_{2}\right) \rightarrow$ $\Phi\left(S_{1}\right)$ by $\phi^{*}(x)=x \circ \phi$. Next assume that $\psi: \Phi\left(S_{2}\right) \rightarrow \Phi\left(S_{1}\right)$ is a homomorphism. Then we claim:

$$
\begin{aligned}
& \text { for all } a \in S_{1} \text {, there exists unique } b \in S_{2} \text { such that } \\
& x(b)=(\psi(x))(a) \text { for all } x \in \Phi\left(S_{2}\right)
\end{aligned}
$$

Assume (6). Then we can define $\bar{\psi}: \mathrm{S}_{1} \rightarrow \mathrm{S}_{2}$ as $\bar{\psi}(\mathrm{a})=\mathrm{b}$. Then

$$
x(\bar{\psi}(a))=(\psi(x))(a) \text { for all } a \in S_{1}, x \in \Phi\left(S_{2}\right)
$$

Next we claim,

$$
\bar{\psi} \text { is a*-homomorphism, } \bar{\psi}(1)=1, \bar{\psi}(0)=0
$$

We now prove (6), (8). Note that the uniqueness of $b$ in (6) follows from Lemma 2.2(3). By Lemma $2.2(1)$ we can assume that $S_{2}$ is a closed submonoid of $\left(K^{n}, \cdot\right)$ 
with zero $0=(0, \ldots, 0)$. Let $x_{i}$ denote the $i^{\text {th }}$ projection of $\mathrm{S}_{2}$ into $\mathrm{K}$. Then by Lemma 2.2, $x_{1}, \ldots, x_{n} \in \Phi\left(S_{2}\right)$ and $\Phi\left(S_{2}\right)=\left\langle x_{1}, \ldots, x_{n}\right\rangle$. By Lemma 2.3, there exist $u_{1}, \ldots, u_{t}, v_{1}, \ldots, v_{t} \in F\left(x_{1}, \ldots, x_{n}\right)$ such that for $b \in K^{n}, b \in s_{2}$ if and only if $u_{i}(b)=v_{i}(b), i=1, \ldots, t$. So $u_{i}\left(x_{1}, \ldots, x_{n}\right)=v_{i}\left(x_{1}, \ldots, x_{n}\right), i=1, \ldots, t$. Hence $u_{i}\left(\psi\left(x_{1}\right), \ldots, \psi\left(x_{n}\right)\right)=v_{i}\left(\psi\left(x_{1}\right), \ldots, \psi\left(x_{n}\right)\right), i=1, \ldots, t$. Thus $u_{i}\left(\left(\psi\left(x_{1}\right)\right)(a), \ldots,\left(\psi\left(x_{n}\right)\right)(a)\right)=v_{i}\left(\left(\psi\left(x_{1}\right)\right)(a), \ldots,\left(\psi\left(x_{n}\right)\right)(a)\right)$ for all $a \in S_{1}$, $i=1, \ldots, t$. So $\left(\left(\psi\left(x_{1}\right)\right)(a), \ldots,\left(\psi\left(x_{n}\right)\right)(a)\right) \in s_{2}$ for all $a \in s_{1}$. Define $\bar{\psi}: S_{1} \rightarrow S_{2}$ as $\bar{\psi}(a)=\left(\left(\psi\left(x_{1}\right)\right)(a), \ldots,\left(\left(\psi\left(x_{n}\right)\right)(a)\right)\right.$. So $\bar{\psi}$ is a *homomorphism, $\bar{\psi}(1)=1$, $\bar{\psi}(0)=0$. Clearly $x_{i}(\bar{\psi}(a))=\left(\left(\psi\left(x_{i}\right)\right)(a)\right.$ for all $a \in S, i=1, \ldots, t$. Since $\Phi\left(s_{2}\right)=\left\langle x_{1}, \ldots, x_{n}\right\rangle,(7)$ and hence $(6)$ is true. It is clear from $(7)$ that

$$
\vec{\psi}^{*}=\psi
$$

Now let $\phi: S_{1} \rightarrow S_{2}$ be a *-homomorphism, $\phi(I)=1, \phi(0)=0$. Then for all $x \in \Phi\left(S_{2}\right)$, $a \in S_{1}$, by $(7)$,

$$
\chi\left(\left(\phi^{*}\right)(a)\right)=\left(\phi^{*}(x)\right)(a)=\chi(\phi(a))
$$

By Lemma 2.2(3),

$$
\overline{\phi^{*}}=\phi
$$

THEOREM 2.4. Let $S_{1}, S_{2}, S_{3}$ be connected d-semigroups with zeros, $\operatorname{dim} S_{i}>0$, $i=1,2,3$. Then

(1) If $\phi: S_{1} \rightarrow S_{2}$ is a *-homomorphism with $\phi(0)=0, \phi(1)=1$, then $\phi^{*}: \Phi\left(\mathrm{S}_{2}\right) \rightarrow \Phi\left(\mathrm{S}_{1}\right)$ is a homomorphism and $\overline{\phi^{*}}=\phi$.

(2) If $i: S_{1} \rightarrow S_{1}$ is the identity map then $i^{*}: \Phi\left(S_{1}\right) \rightarrow \Phi\left(S_{1}\right)$ is the identity map.

(3) If $\psi: \Phi\left(\mathrm{S}_{2}\right) \rightarrow \Phi\left(\mathrm{S}_{1}\right)$ is a homomorphism, then $\bar{\psi}: \mathrm{S}_{1} \rightarrow \mathrm{S}_{2}$ is a *-homomorphism with $\bar{\psi}(0)=0, \bar{\psi}(1)=1$. Moreover $\bar{\psi}^{*}=\psi$.

(4) If $i: \Phi\left(S_{1}\right) \rightarrow \Phi\left(S_{1}\right)$ is the identity map, then $\bar{i}: S_{1} \rightarrow S_{1}$ is the identity map.

(5) If $\phi_{1}: S_{1} \rightarrow S_{2}, \phi_{2}: S_{2} \rightarrow S_{3}$ are *homomorphism with $\phi_{i}(0)=0, \phi_{i}(1)=1$, $i=1,2$, then $\left(\phi_{2} \circ \phi_{1}\right)^{*}=\phi_{1}^{*} \circ \phi_{2}^{*}$. 
(6) If $\psi_{1}: \Phi\left(\mathrm{S}_{2}\right) \rightarrow \Phi\left(\mathrm{S}_{1}\right), \psi_{2}: \Phi\left(\mathrm{S}_{3}\right) \rightarrow \Phi\left(\mathrm{S}_{2}\right)$ are homomorphisms, then $\overline{\psi_{1} \circ \psi_{2}}=\overline{\psi_{2}} \circ \overline{\psi_{1}}$.

(7) $S_{1}$ is *-isomorphic to $S_{2}$ if and only if $\Phi\left(S_{1}\right)$ is isomorphic to $\Phi\left(S_{2}\right)$. PROOF. (1), (3) follow from the equations (6)-(10). (2), (4) are trivial. (7) follows from (2), (4), (5), (6). So we need only prove (5), (6). First we prove (5). Let $\chi \in \Phi\left(S_{3}\right)$. Then for all $a \in S_{1}$,

$$
\begin{aligned}
\left(\left(\phi_{2} \circ \phi_{1}\right)^{*}(x)\right)(a) & =x\left(\phi_{2}\left(\phi_{1}(a)\right)\right) \\
& =\left(\phi_{2}^{*}(x)\right)\left(\phi_{1}(a)\right) \\
& =\left(\phi_{1}^{*}\left(\phi_{2}^{*}(x)\right)\right)(a) \\
& =\left(\left(\phi_{1}^{*} \circ \phi_{2}^{*}\right)(x)\right)(a)
\end{aligned}
$$

So $\left(\phi_{2} \circ \phi_{1}\right)^{*}=\phi_{1}^{*} \circ \phi_{2}^{*}$.

Next we prove (6). Let $a \in S, \quad x \in \Phi\left(S_{3}\right)$. Then by equation (7),

$$
\begin{aligned}
x\left(\overline{\psi_{1} \circ \psi_{2}}(a)\right) & =\left(\left(\psi_{1} \circ \psi_{2}\right)(x)\right)(a) \\
& =\left(\psi_{1}\left(\psi_{2}(x)\right)\right)(a) \\
& =\left(\psi_{2}(x)\right)\left(\bar{\psi}_{1}(a)\right) \\
& =x\left(\bar{\psi}_{2}\left(\bar{\psi}_{1}(a)\right)\right) \\
& =x\left(\bar{\psi}_{2} \circ \bar{\psi}_{1}(a)\right)
\end{aligned}
$$

By Lemma $2.2(3), \overline{\psi_{1} \circ \psi_{2}}=\overline{\psi_{2}} \circ \overline{\psi_{1}}$, proving the theorem.

THEOREM 2.5. Let $\omega_{1}, \ldots, \omega_{n} \in F\left(X_{1}, \ldots, X_{m}\right)$. Let $V=\left\{\left(\omega_{1}(a), \ldots, \omega_{n}(a)\right) \mid a \in K^{m}\right\}$ $\subseteq K^{n}$. Set $S=\bar{v}$. Then $S$ is a closed connected d-submonoid with zero, of $\left(K^{n}, \cdot\right)$. Moreover $\Phi(S) \cong<\omega_{1}, \ldots, \omega_{n}>$.

PROOF. Define $\theta:\left(K^{m}, \cdot\right) \rightarrow\left(K^{n}, \cdot\right)$ as $\theta(a)=\left(\omega_{1}(a), \ldots, \omega_{n}(a)\right)$. Then $\theta$ is a *-homomorphism with image $V$. So $S=\bar{V}$ is connected. Clearly $1=\theta(1), 0=\theta(0) \in S$. Let $x_{i}$ denote the $i^{\text {th }}$ projection of $S$ into $K$. Then by Lemma $2.2, \Phi(S)=$ $\left\langle x_{1}, \ldots, x_{n}\right\rangle$. Let $u, v \in F\left(Y_{1}, \ldots, y_{n}\right)$. Suppose $u\left(x_{1}, \ldots, x_{n}\right)=v\left(x_{1}, \ldots, x_{n}\right)$. Then $u(b)=v(b)$ for all $b \in S$. So 


$$
u\left(\omega_{1}(a), \ldots, \omega_{n}(a)\right)=v\left(\omega_{1}(a), \ldots, \omega_{n}(a)\right) \text { for all } a \in K^{m}
$$

Since $K$ is infinite, $u\left(\omega_{1}, \ldots, \omega_{n}\right)=v\left(\omega_{1}, \ldots, \omega_{n}\right)$ in $F\left(x_{1}, \ldots, x_{m}\right)$. Conversely suppose $u\left(\omega_{1}, \ldots, \omega_{n}\right)=v\left(\omega_{1}, \ldots, \omega_{n}\right)$ in $F\left(x_{1}, \ldots, x_{m}\right)$. Then (1l) is true. So $u(b)=v(b)$ for all $b \in v$. Since $\bar{v}=s, u(b)=v(b)$ for all $b \in S$. So $u\left(x_{1}, \ldots, x_{n}\right)=$ $v\left(x_{1}, \ldots, x_{n}\right)$. It follows that $\Phi(S)=\left\langle x_{1}, \ldots, x_{n}\right\rangle \bumpeq\left\langle\omega_{1}, \ldots, \omega_{n}\right\rangle$.

By Theorem A, Lemmas 2.1, 2.2, Theorems 2.4, 2.5, we have.

THEOREM 2.6. Let $N_{1}$ be the category of connected d-semigroups with zeros of dimension >0 with morphism being *-homomorphisms $\phi$ with $\phi(0)=0, \phi(1)=1$. Let $\mathrm{N}_{2}$ be the category of finitely generated, commutative, idempotent free, totally cancellative semigroups with morphisms being semigroup homomorphisms. Then $(\Phi, *)$ is a contravariant equivalence between $N_{1}$ and $N_{2}$.

THEOREM 2.7. Let $S$ be a closed connected submonoid of $\left(K^{n}, \bullet\right)$ with zero $0=(0, \ldots, 0)$. Then for some $m \in \mathrm{z}^{+}, \omega_{1}, \ldots, \omega_{\mathrm{n}} \in F\left(\mathrm{x}_{1}, \ldots, \mathrm{x}_{\mathrm{m}}\right), \mathrm{s}=\overline{\mathrm{v}}$ where $\mathrm{v}=$ $\left\{\left(\omega_{1}(a), \ldots, \omega_{n}(a)\right) \mid a \in K^{m}\right\}$.

PROOF. Let $x_{i}$ denote the $i^{\text {th }}$ projection of $S$ into $K$. Then by Lemma 2.2, $\Phi(S)=\left\langle x_{1}, \ldots, x_{n}\right\rangle$. By Theorem $A, \Phi(S) \cong\left\langle\omega_{1}, \ldots, \omega_{n}\right\rangle$ for some $m \in z^{+}$, $\omega_{1}, \ldots, \omega_{n} \in F\left(X_{1}, \ldots, X_{m}\right)$ with $X_{i} \leftrightarrow \omega_{i}$. Let $v=\left\{\left(\omega_{1}(a), \ldots, \omega_{n}(a)\right) \mid a \in K^{m}\right\}$ and set $S_{1}=\bar{v}$. Then $I=(I, \ldots, I), 0=(0, \ldots, 0) \in S_{I}$. Let $u, v \in F\left(Y_{1}, \ldots, Y_{n}\right)$. Suppose $u(c)=v(c)$ for all $c \in S$. Then $u\left(x_{1}, \ldots, x_{n}\right)=v\left(x_{1}, \ldots, x_{n}\right)$. So $u\left(\omega_{1}, \ldots, \omega_{n}\right)=$ $v\left(w_{1}, \ldots, w_{n}\right)$. Thus $u(b)=v(b)$ for $a l l b \in v$. Since $\bar{v}=S_{1}, u(b)=v(b)$ for all $b \in S_{1}$. Conversely suppose $u(b)=v(b)$ for all $b \in S_{1}$. Then

$$
u\left(\omega_{1}(a), \ldots, \omega_{n}(a)\right)=v\left(\omega_{1}(a), \ldots, \omega_{n}(a)\right) \text { for all } a \in K^{m}
$$

Since $K$ is infinite, $u\left(\omega_{1}, \ldots, \omega_{n}\right)=v\left(\omega_{1}, \ldots, \omega_{n}\right)$. So $u\left(x_{1}, \ldots, x_{n}\right)=v\left(x_{1}, \ldots, x_{n}\right)$. Thus $u(c)=v(c)$ for all $c \in S$. By Lemma 2.3, $S=S_{1}$.

COROLLARY 2.8. Let $S$ be a closed connected submonoid of $\left(K^{n}, \cdot\right)$ with zero $0=(0, \ldots, 0), \operatorname{dim} S=1$. Then there exist $i_{1}, \ldots, i_{n} \in z^{+}$such that $S=$ $\left\{\left(a^{i_{1}}, \ldots, a^{i_{n}}\right) \mid a \in K\right\}$. Conversely, for any $i_{1}, \ldots, i_{n} \in z^{+}, S$ defined as above is a closed connected submonoid of $\left(K^{n}, \cdot\right)$ with zero $0=(0, \ldots, 0)$ and $\operatorname{dim} S=1$. 
$V=\left\{\left(\omega_{1}(a), \ldots, \omega_{n}(a)\right) \mid a \in K^{m}\right\}$ and $s=\bar{v}$. Let $v_{1}=\left\{\left(\omega_{1}(a, \ldots, a), \ldots, \omega_{n}(a, \ldots, a) \mid\right.\right.$ $a \in K\}, S_{1}=\bar{v}_{1}$. Then $s_{1} \subseteq S$, dim $s_{1}=1$. So $s=s_{1}$. So there exist $i_{1}, \ldots, i_{n} \in z^{+}$ such that $v_{1}=\left\{\left(a^{i_{1}}, \ldots, a^{i} n\right) \mid a \in K\right\}$. Define $\theta: K \rightarrow S$ as $\theta(a)=\left(a^{i_{1}}, \ldots, a^{i}\right)$. Then it is easy to see that $\theta$ is a finite morphism in the usual sense of [5; Section 4.2]. By [5; Proposition 4.2], $\theta(\mathrm{K})=\mathrm{S}$.

THEOREM 2.9. Let $S$ be a connected monoid with zero, dim $S=1$. Then $S$ is *-isomorphic to a semigroup of the type given in Corollary 2.8.

PROOF. By Corollary 1.5, $\mathrm{S}$ is a d-semigroup. We are now done by Lemma 2.2 and Corollary 2.8.

THEOREM 2.10. Let $S$ be a connected semigroup, e, $f \in E(S)$, e $>f$. Then there exists a closed connected subsemigroup $S_{1}$ of $S$ such that $e$ is the identity of $S_{1}$, $f$ is the zero of $S_{1}$ and $\operatorname{dim} S_{1}=1$.

PROOF. We can assume that $e$ is the identity element of $S$ (otherwise we work with eSe). By Lemma 1.1 we are reduced to the case when $f$ is the zero of $S$. By Corollary 1.5, we are reduced to the case when $S$ is also a d-semigroup.

$$
\begin{aligned}
& \Lambda(S)=\{\text { All prime ideals of } S\} \cup\{\emptyset\} . \\
& X(S)=\{S|I| I \in \Lambda(S)\} . \\
& \Omega(S)=\text { Maximal semilattice image of } S .
\end{aligned}
$$

It is easy to see that $(\Lambda(S), \subseteq) \cong(\Lambda(\Omega(S)), \subseteq)$ is a complete lattice. If $S$ is finitely generated, then $\Omega(S)$ is finite and so $(\Lambda(S), \subseteq)$ is a finite lattice.

THEOREM 3.1. Let $S$ be a connected d-semigroup with zero. Define $\alpha: I(S) \rightarrow$ $\Gamma(\Phi(S))$ as $\alpha(I)=\{\chi \mid \chi \in \Phi(S), \chi(a)=0$ for all a $\in I\}$. Define $\beta: \Gamma(\Phi(S)) \rightarrow I(S)$ as $\beta(W)=\{a \mid a \in S, X(a)=0$ for all $\chi \in W\}$. Then $\alpha, \beta$ are inclusion reversing bijections and $B=\alpha^{-1}$. Moreover $\alpha(A(S))=\Lambda(\Phi(S))$.

PROOF. Clearly $\alpha, \beta$ are inclusion reversing. Let $I \in A(S)$. Then $I=e S$ for some $e \in E(S)$. So $\alpha(I)=\{x \mid x \in \Phi(S), x(e)=0\}$. It follows that $\alpha(I) \in \Lambda(\Phi(S))$. Clearly $I \subseteq \beta(\alpha(I))$. We claim that $I=\beta(\alpha(I))$. Suppose not. Then there exists $a \in \beta(\alpha(I))$ such that $a \notin I$. Let a $H f, f \in E(S)$. Then $f \notin I, f \in \beta(\alpha(I))$. So e $\geq$. By Lemma 2.I(2), there exists $\chi \in \Phi(S)$ such that $\chi(f)=1, \chi(e)=0$. So 
$x \in \alpha(I)$ and $f \notin \beta(\alpha(I))$, a contradiction. So

$$
\text { for all } I \in A(S), \quad \alpha(I) \in \Lambda(\Phi(S)) \text { and } \beta(\alpha(I))=I
$$

Let $P \in \Lambda(\Phi(S))$. We claim that $\beta(P) \in A(S)$ and $\alpha(\beta(P))=P$. By Lemma 2.1, this is true for $P=\Phi(S)$. So assume $P \neq \Phi(S)$. Then $F=\Phi(S) \backslash P$ is a subsemigroup of $\Phi(S)$. By Lemma 2.2 we can assume that $S$ is a closed submonoid of some $\left(K^{n}, \cdot\right)$, $0=(0, \ldots, 0) \in S$ and that $\Phi(S)=\left\langle x_{1}, \ldots, x_{n}\right\rangle$ where $x_{i}$ is the $i^{\text {th }}$ projection of S into $K, i=1, \ldots, n$. Let $A=\left\{x_{i} \mid x_{i} \in F\right\}$. Then $\langle A\rangle=F$. Let $e=\left(e_{1}, \ldots, e_{n}\right)$ where $e_{i}=1$ if $x_{i} \in A, e_{i}=0$ if $x_{i} \notin A$. We claim that $e \in S$. Suppose not. Then by Lemma 2.3, there exist $u, v \in F\left(X_{1}, \ldots, X_{n}\right)$ such that $u(a)=v(a)$ for all $a \in S$ and $u(e) \neq v(e)$. Since $u(e)^{2}=u(e)$ and $v(e)^{2}=v(e)$ we can assume that $u(e)=1$, $v(e)=0 . \quad$ Clearly $u\left(x_{1}, \ldots, x_{n}\right)=v\left(x_{1}, \ldots, x_{n}\right)$. Since $u(e)=1, u\left(x_{1}, \ldots, x_{n}\right)$

By Lemma 2.2 and Theorem 2.7, we can assume that $S$ is as in Theorem 2.7, with $e=(1, \ldots, 1), f=(0, \ldots, 0)$. Let $v_{1}=\left\{\left(\omega_{1}(a, \ldots, a), \ldots, \omega_{n}(a, \ldots, a)\right) \mid a \in K\right\}$, $S_{1}=\bar{V}_{1}$. Then e, $f \in S_{1}$, dim $S_{1}=1, S_{1} \subseteq S$. Define $\theta: K \rightarrow S_{1}$ as $\theta(a)=\left(\omega_{1}(a, \ldots, a), \ldots, \omega_{n}(a, \ldots, a)\right)$. Then $\theta$ is $a{ }^{*}$-homomorphism. So $S_{1}$ is connected. This proves the theorem.

\section{POLYTOPES}

If $x \subseteq \mathbb{R}^{n}$, then we let $\mathcal{C}(x)$ denote the convex hull of $x$ (see [4]). The convex hull of a finite set in $\mathbb{R}^{\mathrm{n}}$ is called a polytope [4]. If the vertices of $P$ are rational, then $P$ is said to be a rational polytope. If $X \subseteq P$, then $X$ is said to be a face of $P[4 ; p .35]$ if for all $a, b \in P, \alpha \in(0,1), \alpha a+(1-\alpha) b \in X$ if and only if $a, b \in X$. Let $X(P)$ denote the set of all faces of $P$. Then [4; .21 , $(X(P), \subseteq)$ is a finite lattice. Dimension of $P$ is defined to be the dimension of the affine hull of $P[4 ; p .3]$. Then dimension of $P=$ (length of any maximal chain in $X(P))-1$. Two polytopes $P_{1}, P_{2}$ have the same combinatorial type if $X\left(P_{1}\right) \cong$ $X\left(\mathrm{P}_{2}\right)$ (see [4;p. 38]). By [4;p. 244], every polytope of dimension $\leq 3$ has the same combinatorial type as some rational polytope. However this is not true in general [4: p. 94]. If $u=\left(\alpha_{1}, \ldots, \alpha_{n}\right), v=\left(\beta_{1}, \ldots, \beta_{n}\right) \in \mathbb{R}^{n}$ then let $u \cdot v=$ $\sum_{i=1}^{n} \alpha_{i} \beta_{i}$ denote the inner product of $u$ and $v$. 
Let $S$ be a semigroup. An ideal $I$ of $S$ is said to be semiprime if for all $a \in S, a^{2} \in I$ implies $a \in I$. I is prime if for $a l l a, b \in S$, $a b \in I$ implies $a \in I$ or $b \in I$. Let

$$
\begin{aligned}
& I(S)=\{\text { All ideals of } S\} \\
& A(S)=\{\text { All principal ideals of } S\} \\
& \Gamma(S)=\{\text { All semiprime ideals of } S\} \cup\{\emptyset\}
\end{aligned}
$$

involves only those $x_{1}^{\prime}$ 's with $x_{i} \in F$. So $u\left(x_{1}, \ldots, x_{n}\right) \in F$. Since $v(e)=0$, $v\left(x_{1}, \ldots, x_{n}\right)$ involves at least one $x_{i}$ with $x_{i} \notin F$. So $v\left(x_{1}, \ldots, x_{n}\right) \in P$. This contradiction shows that $e \in S$. Clearly $\chi(e)=1$ for $x \in F, \chi(e)=0$ for $x \in P$. Hence $P=\{x \mid x \in \Phi(S), X(e)=0\}=\alpha(e S)$. By $(12), B(P)=\beta(\alpha(e S))=e S \in A(S)$, $\alpha(\beta(P))=\alpha(e S)=P$. So

$$
\text { for all } P \in \Lambda(\Phi(S)), B(P) \in A(S) \text { and } \alpha(B(P))=P
$$

Clearly

$$
\alpha\left(I_{1} \cup I_{2}\right)=\alpha\left(I_{1}\right) \cap \alpha\left(I_{2}\right) \text { for all } I_{1}, I_{2} \in I(S)
$$

Let $W_{1}, W_{2} \in \Gamma(\Phi(S))$. Then clearly $B\left(W_{i}\right) \subseteq B\left(W_{1} \cap W_{2}\right), i=1,2$. So $\beta\left(W_{1}\right) \cup \beta\left(W_{2}\right) \subseteq B\left(W_{1} \cap W_{2}\right)$. Let a $\in \beta\left(W_{1} \cap W_{2}\right)$. Suppose $a \notin \beta\left(W_{i}\right), i=1,2$.

Then there exist $\theta_{i} \in W_{i}, i=1,2$ such that $\theta_{i}(a) \neq 0, i=1,2$. So $\theta=\theta_{1} \theta_{2} \in W_{1} \cap W_{2}, \quad \theta(a) \neq 0$. So a $\notin B\left(W_{1} \cap W_{2}\right)$, a contradiction. Thus

$$
\boldsymbol{B}\left(\mathrm{W}_{1} \cap \mathrm{W}_{2}\right)=\beta\left(\mathrm{W}_{1}\right) \cup \beta\left(\mathrm{W}_{2}\right) \text { for all } \mathrm{W}_{1}, \mathrm{~W}_{2} \in \Gamma(\Phi(\mathrm{S}))
$$

Clearly $A(S)$ is finite. Let $I \in I(S)$. Then $I=I_{1} \cup I_{2} \cup \ldots \cup I_{k}$ for some $I_{1}, \ldots, I_{k} \in A(S)$. By (12), $\beta\left(\alpha\left(I_{r}\right)\right)=I_{r}, r=1, \ldots, k$. By (14), (15),

$$
\begin{aligned}
\alpha(I) & =\alpha\left(I_{l}\right) \cap \ldots \cap \alpha\left(I_{k}\right) \\
\beta(\alpha(I)) & =\beta\left(\alpha\left(I_{l}\right)\right) \cup \ldots \cup \beta\left(\alpha\left(I_{k}\right)\right) \\
& =I_{l} \cup \ldots \cup I_{k} \\
& =I
\end{aligned}
$$

So

$$
\beta(\alpha(I))=I \text { for all } I \in I(S)
$$

Since $\Phi(S)$ is finitely generated, $\Lambda(\Phi(S))$ is finite. Let $W \in \Gamma(\Phi(S))$. By [2; p. 125, Exercise 9], $W=W_{1} \cap \ldots \cap w_{k}$ for some $w_{1}, \ldots, w_{k} \in \Lambda(\Phi(S))$. Then by (13) 


$$
\begin{aligned}
\alpha\left(\beta\left(W_{r}\right)\right)=W_{r} \text { for } r & =1, \ldots, k \text {. Then by }(14),(15), \\
\beta(W) & =\beta\left(W_{1}\right) \cup \ldots \cup \beta\left(W_{k}\right) \\
\alpha(\beta(W)) & =\alpha\left(\beta\left(W_{1}\right)\right) \cap \ldots \cap \alpha\left(\beta\left(W_{k}\right)\right) \\
& =W_{1} \cap \ldots \cap W_{k} \\
& =W
\end{aligned}
$$

So

$$
\alpha(\beta(W))=W \text { for all } W \in \Gamma(\Phi(S))
$$

By (16) and (17), $\alpha^{-1}=\beta$. By (12), (13), $\alpha(A(S))=\Lambda(\Phi(S))$. This proves the theorem.

REMARK. The classical Hilbert's Nullstellensatz yields a $1-1$ correspondence between the closed subsets of $K^{n}$ and the radical ideals of $K\left[x_{1}, \ldots, x_{n}\right]$. Moreover this restricts to a $1-1$ correspondence between the closed irreducible subsets of $\mathrm{K}^{\mathrm{n}}$ and the prime ideals of $\mathrm{K}\left[\mathrm{x}_{1}, \ldots, \mathrm{X}_{\mathrm{n}}\right]$. Analagously, Theorem 3.1 yields a $1-1$ correspondence between the ideals of a connected d-semigroup with zero $S$ and the semiprime ideals of its character semigroup $\Phi(S)$. Moreover this correspondence restricts to a correspondence between the principal ideals of $S$ and the prime ideals of $\Phi(S)$.

THEOREM 3.2. Let $S$ be a connected d-semigroup with zero. Then $(U(S), \leq) \simeq(E(S), \leq) \simeq(X(\Phi(S)), \subseteq)$.

PROOF. Clearly $(A(S), \subseteq) \cong(U(S), \leq) \cong(E(S), \leq)$. By Theorem 3.1, $(A(S), \subseteq)$ is anti-isomorphic to $(\Lambda(\Phi(S)), \subseteq)$. Clearly $(\Lambda(\Phi(S)), \subseteq)$ is anti-isomorphic to $(X(\Phi(S)), \subseteq)$. This proves the theorem.

Let $Q^{m \times n}$ denote the set of all $m \times n$ matrices over $Q$. The following result is well known. However, we include a proof here for the convenience of the reader.

FACT 3.3. Let $A \in Q^{m \times n}, u=\left(\alpha_{1}, \ldots, \alpha_{m}\right) \in \mathbb{R}^{m}$ such that $u A=0$. Then there exists $v=\left(\beta_{1}, \ldots, \beta_{m}\right) \in Q^{m}$ such that $v A=0$ and for $i=1, \ldots, m, \alpha_{i}>0$ implies $\beta_{i}>0, \alpha_{i}<0$ implies $\beta_{i}<0$.

PROOF. Let $N=\left\{X \mid X \in \mathbb{R}^{m}, X A=0\right\}$ denote the left null space of $A$. Since $A \in Q^{m \times n}$, there exist $u_{1}, \ldots, u_{t} \in Q^{m}$ such that $u_{1}, \ldots, u_{t}$ is a basis of $N$. So 
$u=\sum_{j=1}^{t} \varepsilon_{j} u_{j}$ for some $\varepsilon_{1}, \ldots, \varepsilon_{t} \in \mathbb{R}$. Let $\varepsilon \in \mathbb{R}^{+}, \varepsilon_{1}^{\prime}, \ldots, \varepsilon_{t}^{\prime} \in Q$. Then $\mathrm{v}=\sum_{j=1}^{t} \varepsilon_{j}^{\prime} u_{j} \in N \cap Q^{m}$. For $\Sigma\left|\varepsilon_{j}^{\prime}-\varepsilon_{j}\right|$ small enough, $|u-v|<\varepsilon$. For $\varepsilon$ small enough, the conclusion of the lemma clearly holds.

COROLLARY 3.4 . Let $u_{1}, \ldots, u_{m}, v_{1}, \ldots, v_{n} \in Q^{d}, \alpha_{1}, \ldots, \alpha_{m}, \beta_{1}, \ldots, \beta_{n} \in \mathbb{R}^{+}$ such that $\sum_{i=1}^{m} \alpha_{i} u_{i}=\sum_{j=1}^{n} \beta_{j} v_{j}$. Then there exist $\alpha_{1}^{\prime}, \ldots, \alpha_{m}^{\prime}, \beta_{1}^{\prime}, \ldots, \beta_{n}^{\prime} \in z^{+}$such that $\sum_{i=1}^{m} \alpha_{i}^{\prime} u_{i}=\sum_{j=1}^{n} \beta_{j}^{\prime} v_{j}$.

PROOF. By Fact 3.3 we can choose $\alpha_{1}^{\prime}, \ldots, \alpha_{m}^{\prime}, \beta_{1}^{\prime}, \ldots, \beta_{n}^{\prime} \in Q^{+}$such that $\sum_{i=1}^{m} \alpha_{1}^{\prime} u_{i}=\sum_{j=1}^{n} \beta_{j}^{\prime} v_{j}$. Then for some $s \in z^{+}, \alpha_{i}^{\prime \prime}=s \alpha_{i}, \beta_{j}^{\prime \prime}=s \beta_{j} \in z^{+}, i=1, \ldots, m$, $j=1, \ldots, n . \quad$ Clearly $\sum_{i=1}^{m} \alpha_{i}^{\prime \prime} u_{i}=\sum_{j=1}^{n} \beta_{j}^{\prime \prime} v_{j}$.

THEOREM 3.5. The classes $\{X(S) \mid S$ is a finitely generated, commutative, idempotent-free, totally cancellative smeigroup $\}$ and $\{X(P) \mid P$ is a rational polytope in $\mathbb{R}^{n}$ for some $n \in \mathrm{Z}^{+}$\} are identical to within lattice isomorphisms.

PROOF. Let $\mathrm{S}$ be a finitely generated, commutative, idempotent-free, totally cancellative semigroup. By Theorem A we can assume that $s=\left\langle u_{1}, \ldots, u_{n}>\underline{C}\left(z^{d},+\right)\right.$, $0 \notin \mathrm{S}$. Let $\mathrm{C}=\mathrm{C}\left(u_{1}, \ldots, u_{n}\right)$. By Fact $3.3,0 \notin \mathrm{C}$. So $\mathrm{C} \cap-\mathrm{C}=\emptyset$. By [4; p. I] ], there exists $u \in \mathbb{R}^{d}$ such that $u \cdot a>0$ for $a l l a \in C$. So $u \cdot u_{i}>0, i=1, \ldots, n$. If $v \in \mathbb{R}^{d}$, then $\left|u \cdot u_{i}-v \cdot u_{i}\right|=\left|(u-v) \cdot u_{i}\right| \leq\|u-v\|\left\|u_{i}\right\|$. So for $\|u-v\|$ small enough, $v \cdot u_{i}>0$ for $i=1, \ldots, n$. So, without loss of generality, we can assume that $u \in Q^{d}$, If $a \in S$, then let $\theta(a)=\frac{a}{u \cdot a} \in Q^{d}$. Let $a_{1}, \ldots, a_{k} \in S$, $\alpha_{1}, \ldots, \alpha_{k} \in z^{+}$and set $a=\alpha_{1} a_{1}+\ldots+\alpha_{k} a_{k}$. Then

$$
\begin{aligned}
& \theta(a)=\sum_{i=1}^{k} \beta_{i} \theta\left(a_{i}\right) \in C\left(\theta\left(a_{1}\right), \ldots, \theta\left(a_{k}\right)\right), \\
& \sum_{i=1}^{k} \beta_{i}=1 \text { where } \beta_{i}=\frac{\alpha_{i} a_{i} \cdot u}{a \cdot u}>0, i=1, \ldots, k .
\end{aligned}
$$


So $P=C(\theta(S))=\mathcal{C}\left(\theta\left(u_{1}\right), \ldots, \theta\left(u_{n}\right)\right)$ is a rational polytope. If $X \in X(S)$, then let $\phi(X)=\mathcal{C}(\theta(X)) \subseteq P$. If $F \in X(P)$, then let $\psi(F)=\{a \mid a \in S, \theta(a) \in F\} \subseteq S$.

Let $X \in X(S)$. Let $x, y \in P, \alpha \in(0,1)$ such that $\alpha x+(1-\alpha) y=z \in \phi(X)$. There exist $a_{1}, \ldots, a_{p}, b_{1}, \ldots, b_{q} \in S, c_{1}, \ldots, c_{r} \in X, \alpha_{1}, \ldots, \alpha_{p}, \beta_{1}, \ldots, \beta_{q}$, $\gamma_{1}, \ldots, \gamma_{r} \in(0,1)$ such that $x=\sum \alpha_{i} \theta\left(a_{i}\right), y=\Sigma \beta_{j} \theta\left(b_{j}\right), \quad z=\sum \gamma_{k} \theta\left(c_{k}\right)$, $\Sigma \alpha_{i}=\Sigma \beta_{j}=\Sigma \gamma_{k}=1$. So there exist $\alpha_{1}^{\prime}, \ldots, \alpha_{p}^{\prime}, \quad \beta_{1}^{\prime}, \ldots, \beta_{q}^{\prime}, \gamma_{1}^{\prime}, \ldots, \gamma_{r}^{\prime} \in \mathbb{R}^{+}$such that

$$
\sum \alpha_{i}^{\prime} a_{i}+\sum \beta_{j}^{\prime} b_{j}=\Sigma \gamma_{k}^{\prime} c_{k}
$$

By Corollary 3.4 there exist $\alpha_{1}^{\prime \prime}, \ldots, \alpha_{p}, \beta_{1}^{\prime \prime}, \ldots, \beta_{q}^{\prime \prime}, \gamma_{1}^{\prime \prime}, \ldots, \gamma_{r}^{\prime \prime} \in z^{+}$such that

$$
\sum \alpha_{i} a_{i}+\sum \beta_{j} b_{j}=\sum \gamma_{k} c_{k} \in X
$$

Since $X \in X(S), a_{1}, \ldots, a_{p}, b_{1}, \ldots, b_{q} \in X$. Since $x \in \mathcal{C}\left(\theta\left(a_{1}\right), \ldots, \theta\left(a_{p}\right)\right)$ and $y \in C\left(\theta\left(b_{1}\right), \ldots, \theta\left(b_{q}\right)\right), x, y \in \phi(x)$. Hence $\phi(X) \in X(P)$. Clearly $X \subseteq \psi(\phi(x))$. Let $a \in \psi(\phi(X))$. Then $\theta(a) \in C(\theta(X))$. So there exist $a_{1}, \ldots, a_{p} \in X, \alpha$, $\alpha_{1}, \ldots, \alpha_{p} \in \mathbb{R}^{+}$such that $\alpha_{a}=\sum \alpha_{i} a_{i}$. By Corollary 3.4, there exist $\alpha^{\prime}$, $\alpha_{1}^{\prime}, \ldots, \alpha_{p}^{\prime} \in z^{+}$such that $\alpha^{\prime} a=\sum \alpha_{i}^{\prime} a_{i} \in X$. So $a \in X$. Hence

$$
\text { for all } X \in X(S), \quad \phi(X) \in X(P) \text { and } \psi(\phi(X))=X
$$

Let $F \in X(P)$. By $(18) \psi(F)$ is $\varnothing$ or a subsemigroup of $S$. Let $a, b \in S$ such that $a+b \in \psi(F)$. By (18), $\theta(a+b)=\varepsilon \theta(a)+(I-\varepsilon) \theta(b) \in F$ for some $\varepsilon \in(0,1)$. So $\theta(a), \theta(b) \in F$. Hence $a, b \in \psi(F)$ and $\psi(F) \in X(S)$. Clearly $\phi(\psi(F)) \subseteq F$. Let $x \in F$. Then $x \in P=\phi(S)$. So $x=\sum_{i=1}^{k} \varepsilon_{i} \theta\left(a_{i}\right)$ for some $a_{1}, \ldots, a_{k} \in S, \varepsilon_{1}, \ldots, \varepsilon_{k} \in(Q, 1)$ such that $\sum \varepsilon_{i}=1$. Then $\theta\left(a_{i}\right) \in F, i=1, \ldots, k$. So $a_{1}, \ldots, a_{k} \in \psi(F)$ and $x \in \phi(\psi(F))$. So $\phi(\psi(F))=F$. Hence

$$
\text { for all } F \in X(P), \quad \psi(F) \in X(S) \text { and } \phi(\psi(F))=F
$$

Since $\phi, \psi$ are clearly inclusion preserving, it follows from (19), (20) that $(X(S), \subseteq) \cong(X(P), \subseteq)$.

Conversely let $P \subseteq \mathbb{R}^{m}$ be a rational polytope. Then $P=C\left(a_{1}, \ldots, a_{n}\right)$ for some $a_{1}, \ldots, a_{n} \in Q^{m}$. If $\alpha \in z^{+}$, then clearly $(X(P), \subseteq) \cong(X(\alpha P), \subseteq)$. So we can assume that $a_{1}, \ldots, a_{n} \in z^{m}$. Let $u_{i}=\left(a_{i}, 1\right), i=1, \ldots, n, d=m+1$. Then $P_{1}=C\left(u_{1}, \ldots, u_{n}\right)=$ 
$P \times\{I\} \subseteq \mathbb{R}^{d}$ is a rational polytope and $(X(P), \subseteq) \cong\left(X\left(P_{I}\right), \subseteq\right)$. Let $S=$ $<u_{1}, \ldots, u_{n}>\subseteq z^{d}$. Then $O \notin S$ and $S$ is a finitely generated, commutative, totally cancellative, idempotent-free semigroup. Let $u=(0, I) \in z^{d}$. Then $u \cdot u_{i}=1$, $i=1, \ldots, n$. So $\theta\left(u_{i}\right)=\frac{u_{i}}{u \cdot u_{i}}=u_{i}, i=1, \ldots, n$. By the proof of the first half of this theorem, $(X(S), \subseteq) \cong\left(X\left(P_{1}\right), \subseteq\right)$. This proves the theorem.

If $S$ is a connected d-semigroup with zero, then by [7; Theorem 3.17], $\operatorname{dim} S=$ length of any maximal chain in $E(S)$. By Theorems $2.6,3.2$ and 3.5 we have,

THEOREM 3.6. The classes $\{(E(S), \leq) \mid S$ is a connected d-semigroup with zero, $\operatorname{dim} S>0\}$ and $\left\{(X(P), \subseteq) \mid P\right.$ is a rational polytope in $\mathbb{R}^{n}$ for some $\left.n \in Z^{+}\right\}$are identical to within lattice isomorphisms. Moreover, for any corresponding $S$ and

EXAMPLE 3.7. If $S=\left(K^{4}, \cdot\right)$, then the corresponding polytope $P$ is a tetrahedron

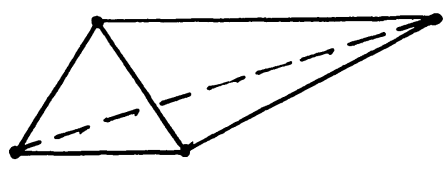

More generally if $S=\left(K^{n}, \cdot\right)$, then the corrsponding polytope $P$ is the $(n-1)$-simplex.

EXAMPLE 3.8. Let $S=\left\{\left(a_{1}, b_{1}, a_{2}, b_{2}, a_{3}, b_{3}\right) \mid a_{i}, b_{j} \in K, a_{i} b_{j}=a_{j} b_{i}, i, j=1,2,3\right\}$. Then by [7; Example 4.7] $\mathrm{S}$ is a closed connected d-submonoid with zero, of $\left(\mathrm{K}^{6}, \cdot\right)$. Moreover dim $S=4$ and $|E(S)|=22$. The corresponding polytope $P$ can be shown to be the triangular prism:

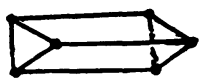

EXAMPLE 3.9. Let $S=\left\{\left(a_{1}, a_{2}, a_{3}, a_{4}, a_{5}, a_{6}\right) \mid a_{1}, \ldots, a_{6} \in K, a_{3}^{2} a_{1}=a_{5} a_{2}^{2}\right.$, $\left.a_{2} a_{5}^{2}=a_{1} a_{4}^{2}, a_{2} a_{4}^{2}=a_{5} a_{3}^{2}\right\}$. Define $\phi: K^{6}+K^{6}$ as

$$
\phi\left(x_{1}, x_{2}, x_{3}, x_{4}, x_{5}, x_{6}\right)=\left(x_{3}^{2} x_{4}^{2} x_{5}^{3}, x_{2}^{2} x_{3}^{2} x_{5}, x_{1} x_{2}^{2} x_{3}, x_{1}^{2} x_{2} x_{4}, x_{1}^{2} x_{4}^{2} x_{5}, x_{6}\right)
$$

Then $\phi\left(K^{6}\right)=S$ and so $S$ is a closed connected d-submonoid with zero, of $\left(K^{6}, \cdot\right)$. Clearly $\operatorname{dim} S=4$ and $|E(S)|=24$. The corresponding polytope $P$ can be shown to be the pentagonal pyramid:

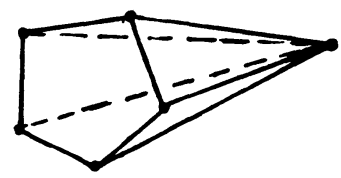


COROLIARY 3.10. Let $S$ be a connected semigroup such that $U(S)$ is the following lattice:

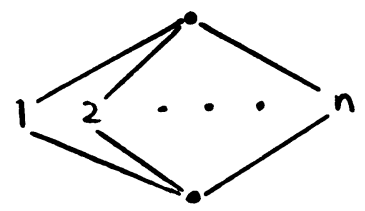

Then $\mathrm{n} \leq 2$.

PROOF. By the proof of Theorem 1.9, we can assume that $S$ is a monoid. Let $\mathrm{S}_{1}$ be a maximal connected d-submonoid with zero, of $\mathrm{S}$. Then by Theorem $1.8(3)$ $E\left(S_{1}\right) \cap J \neq \emptyset$ for all $J \in U(S)$ and $\operatorname{dim} S_{1}=2$. By Theorem 3.6 , the polytope $P$ corresponding to $S_{1}$ has dimension 1 . So $P$ is the line

So $\left|E\left(S_{1}\right)\right|=4$. Thus $|U(S)| \leq\left|E\left(S_{1}\right)\right|=4$. Hence $n \leq 2$.

EXAMPLE 3.11. $M_{2}(K)$ and $\left(K^{2}, \cdot\right)$ show that $n$ can be 1 or 2 in Corollary 3.10 .

\section{SEMILATTICES}

As usual, by a semilattice, we mean a commutative, idempotent semigroup. LEMMA 4.1. Let $\Omega$ be a finite semilattice. Then $|X(\Omega)|=|\Omega|+1$. PROOF. We prove by induction on $|\Omega|$. If $|\Omega|=1$ this is clear. So assume $|\Omega|>1$. Let $\alpha$ be a maximal element of $\Omega$. Then $\{\alpha\} \in X(\Omega)$. Define $\phi: X(\Omega) \rightarrow X\left(\Omega_{1}\right)$ as $\phi(F)=F \cap \Omega_{1}$. Let $F_{1} \in X\left(\Omega_{1}\right)$ and set $P_{1}=\Omega_{1} \backslash F_{1}$. Let $p \in P_{1}$. We claim that $\alpha p \in P_{1}$. Otherwise $f=\alpha p \in F_{1}$. Then $f=p f \in P_{1}$, a contradiction. So $\alpha P_{1} \subseteq P_{1}$. If $F_{1} \in X(\Omega)$, then $\phi\left(F_{1}\right)=F_{1}$. Suppose not. Then $\alpha F_{1} \nsubseteq P_{1}$. So there exists $f_{1} \in F_{1}$ such that $\alpha f_{1} \in F_{1}$. Now we claim that $F_{1} \cup\{\alpha\} \in X(\Omega)$. Otherwise $\alpha F_{1} \nsubseteq F_{1}$. So $\alpha f_{2} \in P_{1}$ for some $f_{2} \in F_{1}$. So $\alpha f_{1} f_{2}=\left(\alpha f_{1}\right) f_{2} \in F_{1}$ and $\alpha f_{1} f_{2}=\left(\alpha f_{2}\right) f_{1} \in P_{1}$, a contradiction. So $F_{1} \cup\{\alpha\} \in X(\Omega), \phi\left(F_{1} \cup\{\alpha\}\right)=F_{1}$. Thus $\phi$ is surjective. Let $F_{I} \in X\left(\Omega_{1}\right), F_{1} \neq \varnothing, F, G \in X(\Omega), \phi(F)=F_{I}=\phi(G), F \neq G$. We can assume that $\alpha \in F, \alpha \notin G$. So $G=F_{1}, F=F_{1} \cup\{\alpha\}$. Since $\alpha \in F$, $\alpha F \subseteq F$. So $\alpha F_{1} \subseteq F_{1}$. 
Since $\alpha \notin G$, $\alpha G \subseteq \Omega \backslash G$. So $\alpha F_{1} \subseteq \Omega_{1} \backslash F_{1}$, a contradiction. Thus $\left|\phi^{-1}\left(F_{1}\right)\right|=1$. Clearly $\phi^{-1}(\varnothing)=\{\varnothing,\{\alpha\}\}$. So $|X(\Omega)|=\left|X\left(\Omega_{1}\right)\right|+1=\left|\Omega_{1}\right|+1+1=|\Omega|+1$.

If $\Omega$ is a semilattice, then let $Y(\Omega)$ denote the semilattice of all homomorphisms of $\Omega$ into $\Omega_{0}=\{0,1\}$. Then clearly $Y(\Omega) \cong(X(\Omega), \cap)$. Let $Y^{*}(\Omega)=Y(\Omega) \backslash\{1,0\}$. Then $y^{*}(\Omega)$ may or may not be a subsemilattice of $y(\Omega)$.

LEMMA 4.2. Let $\Omega$ be a finite semilattice. Then $y^{*}(y(\Omega))$ is a semilattice and $\Omega \cong y^{*}(y(\Omega))$.

PROOF. Define $\theta: \Omega \rightarrow Y(Y(\Omega))$ as $\theta(\alpha)(f)=f(\alpha)$. Then $\theta$ is a homomorphism. Clearly $\theta(\alpha)(1)=1, \theta(\alpha)(0)=0$. So $\theta(\alpha) \in y^{*}(y(\Omega))$. We claim that $\theta$ is injective. We can assume that $\Omega \subseteq \Omega_{0} \times \ldots \times \Omega_{0}$. Let $f_{i}$ denote the $i^{\text {th }}$ projection of $\Omega$ into $\Omega_{0}$. Then $f_{i} \in Y(\Omega)$. Let $\alpha, \beta \in \Omega$ such that $\theta(\alpha)=\theta(\beta)$. Then $\theta(\alpha)\left(f_{i}\right)=\theta(\beta)\left(f_{i}\right)$ for all $i$. So $f_{i}(\alpha)=f_{i}(\beta)$ for all $i$. So $\alpha=\beta$. By Lemma 4.1, $\left|y^{*}(y(\Omega))\right|=|\Omega|$. Hence $\Omega \cong y^{*}(y(\Omega))$.

COROLLARY 4.3. Let $\Omega_{1}, \Omega_{2}$ be finite semilattice such that $\left(X\left(\Omega_{1}\right), \subseteq\right) \cong\left(X\left(\Omega_{2}\right), \subseteq\right)$. Then $\Omega_{1} \cong \Omega_{2}$.

COROLLARY 4.4. Let $\Omega$ be a finite semilattice such that $y^{*}(\Omega)$ is a semilattice. Then $y\left(y^{*}(\Omega) \cong \Omega\right.$.

PROOF. Let $\Omega_{1}=y^{*}(\Omega)$. Then by Lemma 4.2 ,

$$
y^{*}\left(y\left(\Omega_{1}\right)\right) \cong \Omega_{1}=y^{*}(\Omega) \text {. }
$$

So $y\left(y\left(\Omega_{1}\right)\right) \cong y(\Omega)$. Again by Lemma 4.2 ,

$$
y\left(\Omega_{1}\right) \cong y^{*}\left(y\left(y\left(\Omega_{1}\right)\right)\right) \cong y^{*}(y(\Omega)) \cong \Omega
$$

If $S$ is a finitely generated semigroup and if $\Omega$ is the maximal semilattice image of $S$, then clearly $\Omega$ is finite and $(X(S), \subseteq) \cong(X(\Omega), \subseteq)$. By Theorem 3.5 , Lemma 4.2, Corollaries $4.3,4.4$, we have.

THEOREM 4.5. (I) Let $(L, V, \Lambda)$ be a finite lattice. Then $L \cong X(P)$ for some rational polytope $P$ if and only if $\Omega=y^{*}(L, \Lambda)$ is a semilattice and $\Omega$ is 
isomorphic to the maximal semilattice image of some finitely generated, commutative, idempotent free, totally cancellative semigroup.

(2) Let $\Omega$ be a finite semilattice. Then $\Omega$ is the maximal semilattice image of some finitely generated, commutaitve, idempotent free, totally cancellative semigroup if and only if $(X(\Omega), \subseteq)$ is isomorphic to $(X(P), \subseteq)$ for some rational polytope $P$.

If $P$ is a polytope, call $X(P)$, the face lattice of $P$. By a theorem of Tarski (see [4; p. 91]), the enumeration problem for face lattices of polytopes is solvable. However, for rational polytopes the problem is not yet solved [4; p. 92]. By. Theorem 4.5, we have,

THEOREM 4.6. The enumeration problem for face lattices of rational polytopes is solvable if and only if the enumeration problem for maximal semilattice images of finitely generated, commutative, idempotent-free totally cancellative semigroups, is solvable.

\section{REFERENCES}

1. BOREL, A. Linear algebraic groups, W. A. Benjamin, N. Y., 1969.

2. CLIFFORD, A. H. and G. B. PRESTON The algebraic theory of semigroups, vol. I, Amer. Math. Soc., Providence, R. I., 1961.

3. GRILLET, P. A. The free envelope of a finitely generated commutative semigroup, Trans. AMS 149(1970) 665-682.

4. GRÜNBAUM, B. Convex polytopes, Interscience Publishers, John Wiley \& Sons Ltd., 1967.

5. HUMPHREYS, J. E. Linear algebraic groups, Springer-Verlag, 1975.

6. PUTCHA, M. S. On linear algebraic semigroups, Trans. Amer. Math. Soc. 259 (1980) $457-469$.

7. PUTCHA, M.S. On linear algebraic semigroups II, Trans. Amer. Math. Soc. 259 (1980) 471-491.

8. PUTCHA, M. S. Semilattice decompositions of semigroups, Semigroups Forum 6 (1973) 12-34. 


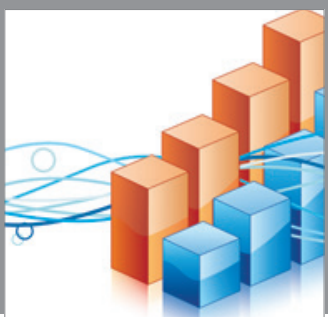

Advances in

Operations Research

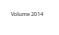

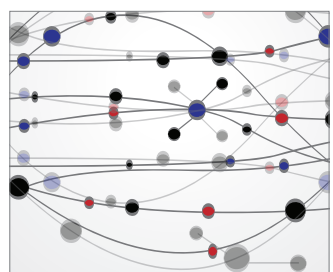

\section{The Scientific} World Journal
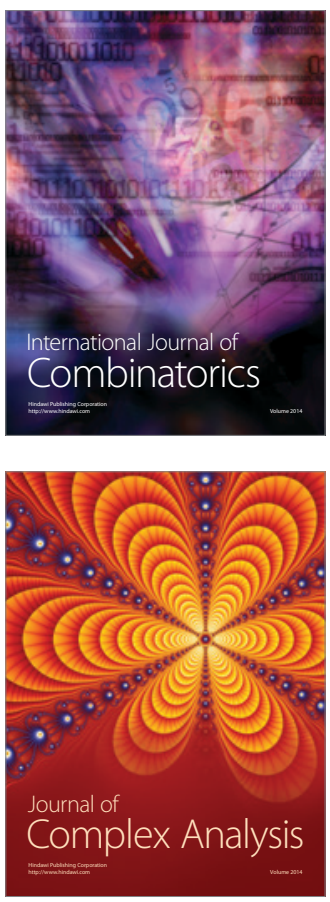

International Journal of

Mathematics and

Mathematical

Sciences
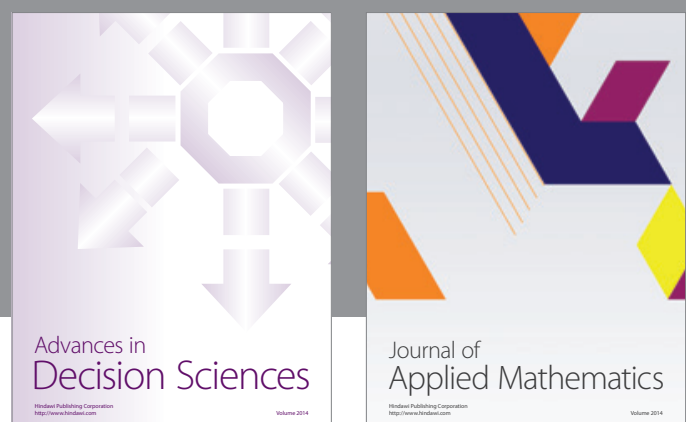

Journal of

Applied Mathematics
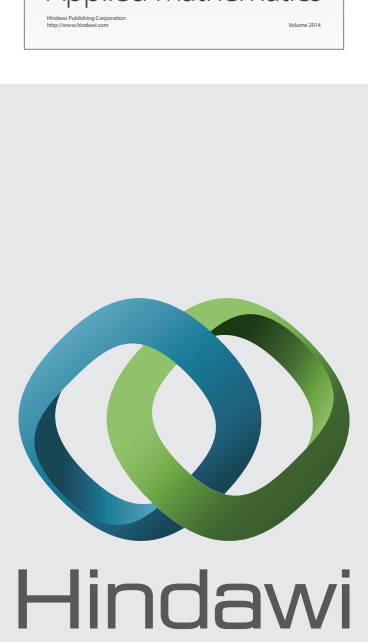

Submit your manuscripts at http://www.hindawi.com
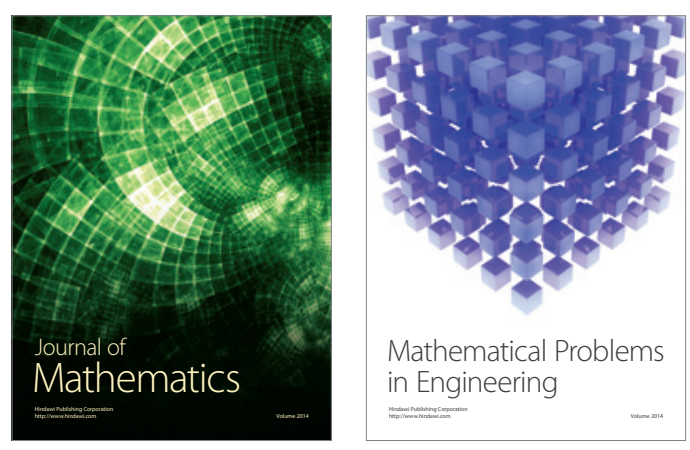

Mathematical Problems in Engineering
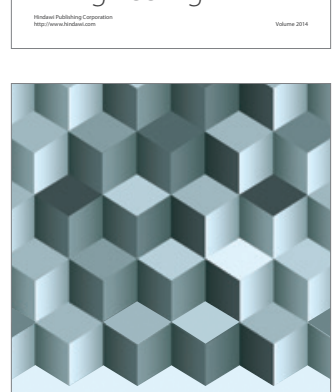

Journal of

Function Spaces
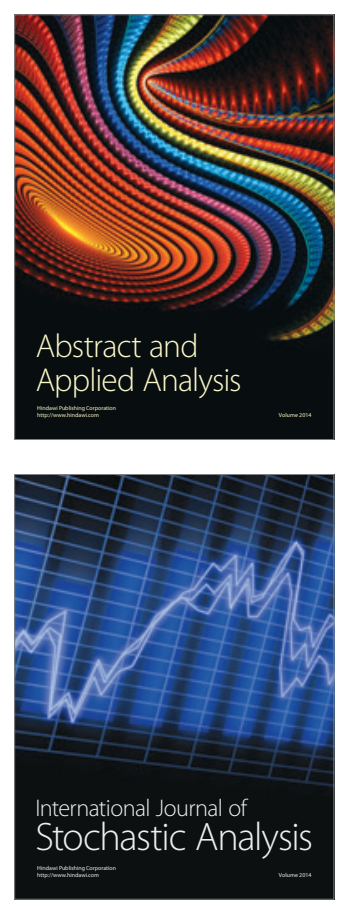

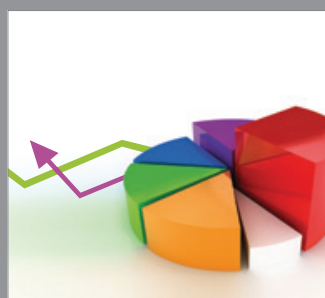

ournal of

Probability and Statistics

Promensencen
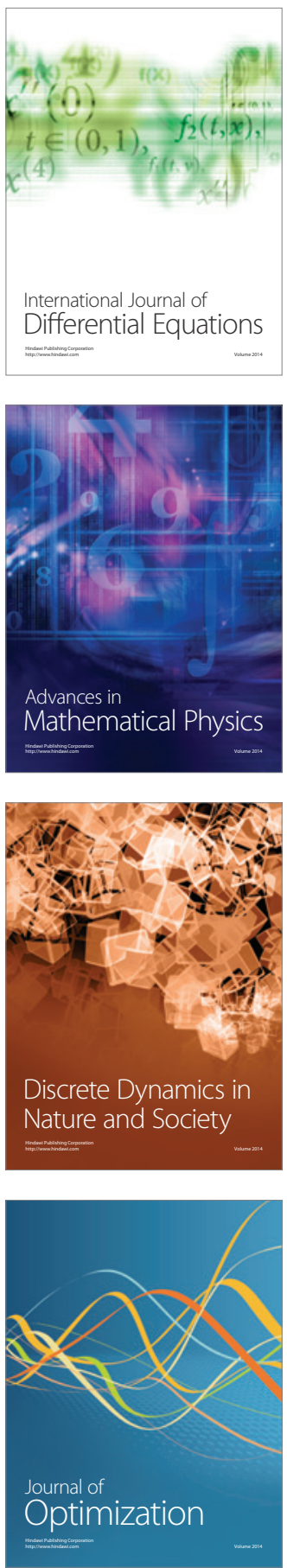